\title{
ESTRUTURA E FLORÍSTICA DE UM REMANESCENTE DE CAATINGA ARBÓREA EM JUVENÍLIA, NORTE DE MINAS GERAIS, BRASIL
}

\author{
Rubens Manoel dos Santos ${ }^{1}$, Ana Carolina Maioli Campos Barbosa ${ }^{2}$, Hisaias de Souza Almeida ${ }^{3}$, \\ Fábio de Almeida Vieira ${ }^{4}$, Paola Ferreira Santoss, Douglas Antônio de Carvalho, \\ Ary Teixeira de Oliveira-Filho ${ }^{7}$
}

(recebido: 9 de fevereiro de 2009; aceito: 29 de março de 2011)

\begin{abstract}
RESUMO: Devido à heterogeneidade fitofisionômica da vegetação na região Norte Mineira, a estrutura e riqueza de seus remanescentes são consideravelmente variáveis, e, embora pouco conhecida, trata-se de uma região amplamente explorada economicamente. Objetivou-se, com este estudo, caracterizar a estrutura e florística de uma área de caatinga arbórea, localizada em

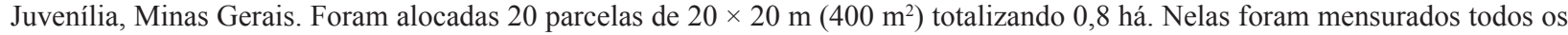
indivíduos com CAP (circunferência à altura do peito) $\geq 10 \mathrm{~cm}$. Para complementar a listagem florística do componente arbustivoarbóreo, foram realizadas quatro visitas para coleta de material botânico entre janeiro e dezembro de 2003 . A composição florística foi representada por 64 espécies arbóreas, destas, 47 foram amostradas no levantamento fitossociológico. A diversidade da área foi de $H^{\prime}=2,90$ e $J^{\prime}=0,76$, com dominância das espécies Anadenanthera colubrina, Poincianella pluviosa e Myracrodruon urundeuva que apresentaram os maiores valores de Dominância: 26,3, 13,1 e 20,3\%; Densidade: 11,2, 16,6 e 10,1\%; Frequência: 6,2, 6,5 e $5,5 \%$ e Valor de Importância: 14,6, 12,1 e 12,0\%, respectivamente. A distribuição diamétrica da comunidade tendeu à normalidade, com menor quantidade de indivíduos nas classes diamétricas menores e maiores. A comunidade estudada mostra padrões estruturais e florísticos semelhantes a outros remanescentes de florestas deciduais em bom estado de conservação, dentro e fora do bioma Caatinga, embora tenha sido evidenciada a presença de interferências antrópicas na área.
\end{abstract}

Palavras-chave: Distribuição diamétrica, diversidade, dominância ecológica.

\section{STRUCTURE AND FLORISTICS OF A REMNANT OF ARBOREOUS CAATINGA IN JUVENÍLIA, NORTHERN MINAS GERAIS, BRAZIL}

\begin{abstract}
Due to the phytophysiognomic heterogeneity of the northern Minas Gerais region, the structure and richness of local vegetation remnants vary considerably, and regardless of the fact that the region is yet little known, it has been widely explored economically. The objective of this study is to characterize the structure and floristics of a fragment of Arboreous Caatinga located in Juvenilia, Minas Gerais. Twenty $20 \times 20 \mathrm{~m}$ plots $\left(400 \mathrm{~m}^{2}\right)$ were allocated, to a total of $0.8 \mathrm{ha}$, and all individuals in them with a CAP (circumference at breast height) $\geq 10 \mathrm{~cm}$ were recorded. To complement the floristic listing of shrub and tree components, four visits were undertaken to collect botanical material, between January and December 2003. The floristic composition was represented by 64 arboreous species, out of which 47 were sampled in the phytosociological survey. The local diversity was $H^{\prime}=$ 2.90 and $J^{\prime}=0.76$, with a dominance of species Anadenanthera colubrina, Poincianella pluviosa and Myracrodruon urundeuva, respectively with Dominance: $26.3 \%, 13.1 \%$ and $20.3 \%$; Density: $11.2 \%, 16.6 \%$ and $10.1 \%$; Frequency: $6.2 \%, 6.5 \%$ and $5.5 \%$ and Importance Value: $14.6 \%, 12.1 \%$ and $12.0 \%$. The diameter distribution of the community tended to normality, with less individuals in smaller and larger diameter classes. The structural and floristic patterns of the community in question were found to be similar to other reasonably preserved remnants of deciduous forest, inside and outside the Caatinga biome, despite the evidence of local anthropic intervention.
\end{abstract}

Key words: Diameter distribution, diversity, ecological dominance.

\footnotetext{
${ }^{1}$ Biólogo, Professor Dr. em Engenharia Florestal - Departamento de Ciências Florestais/DCF - Universidade Federal de Lavras/UFLA - Cx. P. 3037 37200-000 - Lavras, MG - rubensmanoel@dcf.ufla.br

${ }^{2}$ Engenheira Florestal, Professora Dra. em Engenharia Florestal - Departamento de Ciências Florestais/DCF - Universidade Federal de Lavras/UFLACx. P. 3037 -37200-000 - Lavras, MG - accampo@gmail.com

${ }^{3}$ Biólogo, Doutorando em Engenharia Florestal - Departamento de Ciências Florestais/DCF - Universidade Federal de Lavras/UFLA - Cx. P. 3037 37200-000 - Lavras, MG - hisaias37@gmail.com

${ }^{4}$ Biólogo, Professor Dr. em Engenharia Florestal - Departamento de Engenharia Florestal - Universidade Federal do Rio Grande do Norte - Unidade de Ciências Agrárias - RN 160, Km 03 - Cx. P. 7 - 59280-000 - Macaiba, RN - vieirafa@yahoo.com.br

${ }^{5}$ Bióloga - Faculdade de Saúde Ibituruna-Santa Casa - Avenida Nice, N 99, Ibituruna - 39400-089 - Montes Claros, MG

${ }^{6}$ Agrônomo, Professor Dr. em Biologia Vegetal - Departamento de Biologia - Universidade Federal de Lavras/UFLA - Cx. P. 3037 - $37200-000$ Lavras, MG - douglasc@dcf.ufla.br

${ }^{7}$ Engenheiro Florestal, Professor Dr. em Ecologia e Fitogeografia - Departamento de Botânica - Instituto de Ciências Biológicas - Universidade Federal de Minas Gerais/UFMG - Cx. P. 486 - 31270-910 - Belo Horizonte, MG - ary.oliveira.filho@gmail.com
}

Cerne, Lavras, v. 17, n. 2, p. 247-258, abr./jun. 2011 


\section{INTRODUÇÃO}

A Caatinga brasileira encontra-se na região nordeste do Brasil, ocupando cerca de oito estados (PRADO, 2003) e representa o maior núcleo de florestas deciduais na América Latina (PRADO; GIBBS, 1993), com mais de $800.000 \mathrm{~km}^{2}$ (PRADO, 2003). Devido a sua diversidade climática (SAMPAIO, 1995), edáfica e geomorfológica (AB'SABER, 1995), a Caatinga é composta por um mosaico de formações savânicas, além de florestas deciduais e úmidas (QUEIROZ, 2006; RODAL, 2008). Apesar de sua boa representatividade entre os biomas brasileiros, pouco se conhece sobre a sua flora, além disso, muitos trabalhos que visam ao levantamento de espécies na Caatinga encontram-se concentrados em determinadas regiões, principalmente ao norte do bioma (QUEIROZ, 2006; TABARELLI; SILVA, 2003).

No Norte do Estado de Minas Gerais, encontra-se a fronteira sul para o bioma da Caatinga (QUEIROZ, 2006). Esta região apresenta clima de estacionalidade marcante, com isoietas próximas a $1000 \mathrm{~mm}$ e que está incluído no "polígono das secas" (PRADO, 2003). Tais características conferem à vegetação sobrevivência ligada à deficiência hídrica adaptada a um clima severo, com baixa precipitação anual distribuída em um curto período do ano (entre três e cinco meses) (SANO; ALMEIDA, 1998). Esta região possui uma variedade de fitofisionomias da Caatinga em transição com o Cerrado, sendo a caatinga arbórea uma das mais características, florística e fisionomicamente (FERNANDES, 2003; SANTOS, 2009).

No Norte de Minas e em outras regiões, a Caatinga arbórea ainda é pouco estudada e, de acordo com Tabarelli e Silva (2003), esta região destaca-se por ser uma área de prioridade para a conservação da diversidade biológica da Caatinga. Apesar destas particularidades, a Caatinga arbórea no Norte de Minas Gerais vem sofrendo intensa exploração dos recursos naturais, principalmente para dar lugar à prática da agropecuária e à extração de madeira para carvão (observação pessoal dos autores). Atualmente ainda podem ser encontrados remanescentes desta formação em Juvenília e em outros municípios do Norte do Estado, áreas que merecem ações urgentes de preservação.

Neste contexto, visando contribuir para ações futuras de preservação e manejo adequados de áreas de Caatinga arbórea, objetivou-se, com este estudo, determinar a estrutura e a florística de um remanescente de caatinga arbórea, localizada em Juvenília, Norte de Minas Gerais.

\section{MATERIAL E MÉTODOS}

\section{1 Área do estudo}

A área de estudo compreende um remanescente florestal com área de 80 ha, localizado na fazenda Vale Verde $\left(14^{\circ} 24^{\prime} 88^{\prime}\right.$ " S e $44^{\circ} 09^{\prime} 79^{\prime}$ ' W, com altitude de $658 \mathrm{~m}$ ) no município de Juvenília, extremo Norte de Minas Gerais (Figura 1a). O tipo climático segundo o sistema de classificação de Köppen é o tropical semi-árido (Bsh), com verões quentes e secos. A temperatura média anual se encontra em torno dos $23^{\circ} \mathrm{C}$ e a precipitação média é de aproximadamente $1.000 \mathrm{~mm} \cdot \mathrm{ano}^{-1}$, com chuvas concentradas nos meses de novembro a janeiro (informações provenientes da estação metrológica localizada no município de Juvenília). O solo no fragmento foi classificado em Argissolos Vermelho-Amarelos Eutrófico (PVAe) e Nitossolos Vermelhos Eutrófico (NVe), (EMPRESA BRASILEIRA DE PESQUISA AGROPECUÁRIA EMBRAPA, 1997). A topografia é plana com pequenas elevações nas áreas de afloramento de calcário.

De acordo com Santos (2009), o fragmento está inserido em uma região com predominância da fitofisionomia caatinga arbórea (floresta estacional decidual), todavia, para ratificar esta classificação fitofisionômica, foi testada a semelhança florística entre a área de estudo e cinco das principais formações florestais brasileiras. Deste modo, foi realizado um dendrograma com uma matriz biótica contendo as espécies amostradas neste trabalho bem como as espécies indicadoras das fitofisionomias: (a) Caatinga Arbórea (SANTOS, 2009); (b) Caatinga do Cristalino (SANTOS, 2009); (C) Florestas Estacionais Deciduais extra-Caatinga (ALMEIDA; MACHADO, 2007; SANTOS, 2009); (d) Floresta Estacional Semidecidual Submontana e Montana (OLIVEIRA-FILHO; FONTES, 2000); (e) Floresta Estacional Semidecidual de baixa altitude (OLIVEIRA-FILHO; FONTES, 2000) (f) Floresta Estacional Semidecidual Altimontana (OLIVEIRAFILHO; FONTES, 2000) e (g) Grupo de espécies de ampla ocorrência "Supertramps" (OLIVEIRA-FILHO; FONTES, 2000). Foram utilizados o índice de dissimilaridade de 1-Sorense e a técnica de ligação de média ponderada UPGMA (KENT; COKER, 1992).

Embora o fragmento encontra-se em bom estado de conservação, foram observados sinais evidentes de impactos localizados, como exploração seletiva de madeira e trilhas provocadas pela constante penetração de gado e caprinos em busca de alimento e abrigo. A paisagem local é dominada por pastagem e por áreas abandonadas em processo de regeneração natural.

Cerne, Lavras, v. 17, n. 2, p. 247-258, abr./jun. 2011 


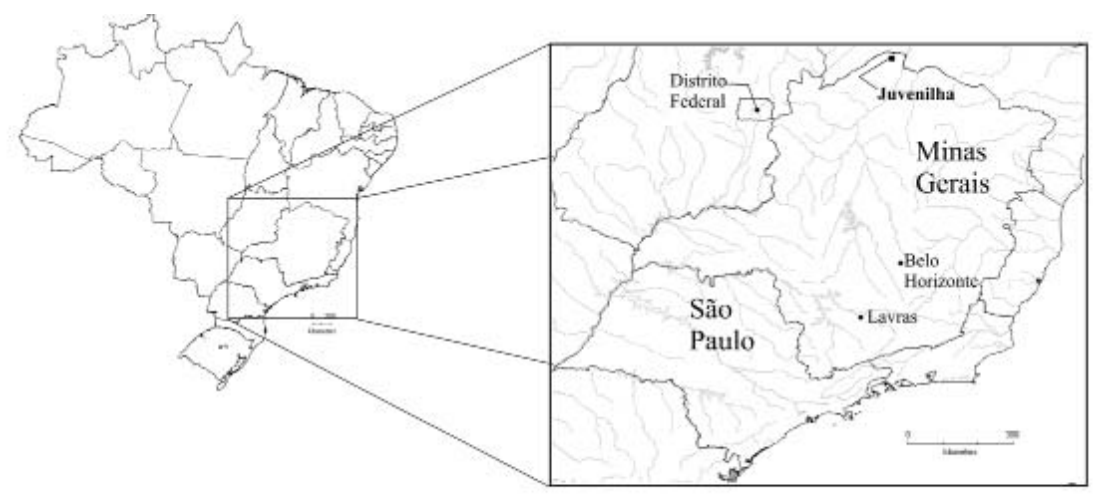

(a)

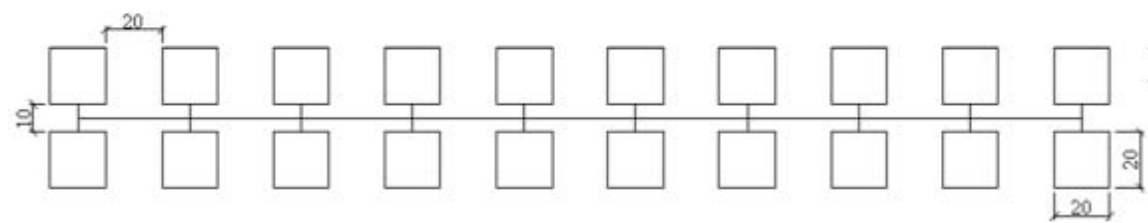

(b)

Figura 1 - Localização geográfica (a) e disposição das parcelas (b) na área do remanescente estudado "Vale Verde", em Juvenília, Norte do Estado de Minas Gerais.

Figure 1 - Geographic location (a) and plot arrangement (b) in the relevant 'Vale Verde' remnant, in Juvenilia, northern Minas Gerais state.

\subsection{Levantamento florístico}

O levantamento florístico das espécies arbóreas ocorreu entre janeiro e dezembro de 2003, com quatro visitas na área, sendo realizadas expedições de coleta na área a cada 90 dias. Foram realizadas incursões ao interior do fragmento, através de caminhadas aleatórias que variavam de quatro a cinco horas, para caracterizar a flora local e coletar material botânico reprodutivo. A herborização do material botânico foi realizada no Herbário Montes Claros (HMC) da Universidade Estadual de Montes Claros (MG) e a coleção testemunha incorporada ao acervo do mesmo. A identificação do material botânico foi realizada com a utilização de coleções botânicas já existentes no HMC, por meio de consulta a literatura especializada e a especialistas. O sistema de classificação adotado foi o do Angiosperm Phylogeny Group - APG II (2003).

\subsection{Levantamento estrutural}

Para o levantamento estrutural, foram alocadas 20 parcelas de $20 \times 20 \mathrm{~m}\left(400 \mathrm{~m}^{2}\right)$, distribuídas sobre um transecto paralelo ao maior eixo do fragmento, sendo que a cada 20 metros, foram alocadas duas parcelas, distantes 10 metros entre si, totalizando 0,8 ha amostrados (Figura 1b). Os vértices das parcelas foram marcados com canos de PVC e ligados por fitilho.
Foram mensurados todos os indivíduos com CAP (circunferência à altura do peito) $\geq 10 \mathrm{~cm}(\mathrm{DAP} \geq 3,7$ $\mathrm{cm}$ ). Para os indivíduos que perfilharam acima do solo e abaixo da altura do peito $(1,30 \mathrm{~m})$, também foi utilizado o mesmo critério de inclusão, porém foram adotados os procedimentos indicados por Scolforo e Mello (1997): (a) indivíduos bifurcados a $1,30 \mathrm{~m}$ foram medidos logo abaixo da bifurcação; (b) para indivíduos bifurcados abaixo de $1,30 \mathrm{~m}$, mediram-se todos os CAP's e o CAP total foi determinado por meio da equação: $C t=\sqrt{\left(c_{1}{ }^{2}+c_{2}{ }^{2}+\ldots c_{i}{ }^{n}\right)}$ em que ' $C t$ ' é a circunferência total a ser calculada e ' $c$ ' as respectivas CAPs mensuradas em campo. Os indivíduos amostrados foram marcados com plaquetas de alumínio numeradas, para futuras medições de dinâmica.

Para cada indivíduo, foram anotados valores de CAP e altura, utilizando-se os estágios da vara de poda graduados, e coletado material botânico reprodutivo para posterior confirmação ou identificação ex-situ. Para descrever a estrutura horizontal da floresta, foram calculados os seguintes parâmetros estruturais: densidade absoluta (DA), densidade relativa (DR), frequência absoluta (FR), frequência relativa (FR), dominância absoluta (DOA), dominância relativa (DOR) e valor de importância (VI) (MUELLER-DOMBOIS; ELLEMBERG, 1974), além dos índices de diversidade de Shannon $\left(H^{\prime}\right)$ e equabilidade de Pielou ( $\left.J^{\prime}\right)$ (MARTINS, 1993).

Cerne, Lavras, v. 17, n. 2, p. 247-258, abr./jun. 2011 


\subsection{Distribuição diamétrica}

A partir dos dados estruturais, obteve-se a distribuição dos indivíduos em classes de diâmetro para a comunidade, e para os indivíduos das espécies cujo somatório de seus respectivos VIs fosse igual ou próximo a um terço ( $\cong 33 \%)$ do VI da comunidade. As distribuições dos indivíduos por classes de diâmetro, com intervalo de classe de amplitudes crescentes, foram um recurso utilizado para compensar o forte decréscimo da densidade nas classes de tamanho maiores (BOTREL et al., 2002). Estes intervalos permitem melhor representação das classes diamétricas maiores e de baixa densidade (OLIVEIRA-FILHO et al., 1998).

\section{RESULTADOS E DISCUSSÃO}

Florística - Foram identificadas 64 espécies arbóreas, num total de 52 gêneros e 26 famílias amostradas (Tabela 1). As famílias de maior número de espécies foram Fabaceae com 26; Malvaceae com cinco; Bignoniaceae e Anacardiaceae com 3; seguidas por Cactaceae, Euphorbiaceae, Meliaceae, Rubiaceae e Rutaceae com 2 espécies cada, que juntas representaram $73 \%$ da flora amostrada. Dentre as demais famílias, aproximadamente $65 \%$, foram representadas por uma única espécie. A nível genérico, Machaerium foi o mais representado com 6 espécies, seguido por Handroanthus, Albizia, Piptadenia, Platymiscium e Senna com 2 espécies cada, totalizando cerca de $28 \%$ das espécies amostradas.

A análise de agrupamento indica a formação de dois grupos florísticos (Figura 2). O grupo A, que contém o fragmento Vale Verde (este trabalho) e as espécies indicadoras da fitofisionomia Caatinga Arbórea, o que corrobora com a classificação de Santos (2009). Já o segundo grupo, B, é composto pelas fitofisionomias estacionais extra-Caatinga, e pode ser separado em dois subgrupos, o $\mathrm{B} 1$, formado pelas áreas de Florestas Estacionais Deciduais e espécies de ampla ocorrência, o que já era esperado ao se considerar o caráter transicional da posição geográfica destas áreas (AB'SABER, 1995) e o B2, formado pelas fitofisionomias de Floresta Estacional Semideciduais de baixa altitude, submontana e montana. Além destes grupos, foi possível observar que as fitofisionomias de Caatinga de áreas do Cristalino e a Floresta Estacional Semidecidual de Altitude compartilharam poucas espécies indicadoras com as demais formações.

Tabela 1 - Espécies amostradas nos levantamentos florístico $\left(^{*}\right)$ e estrutural, com seus respectivos parâmetros fitossociológicos em um remanescente de Caatinga Arbórea em Juvenília, MG. DA - Densidade Absoluta; DR - Densidade Relativa; FA - Frequência Absoluta; FR - Frequência Relativa; DoA - Dominância Absoluta; DoR - Dominância Relativa e VI = Valor de Importância.

Table 1 - Species sampled in the floristic (*) and structural surveys, with the respective phytosociological parameters in a remnant of Arboreous Caatinga in Juvenília, MG. DA - Absolute Density; DR - Relative Density; FA - Absolute Frequency; FR - Relative Frequency; DoA - Absolute Dominance; DoR - Relative Dominance and VI = Importance Value.

\begin{tabular}{|c|c|c|c|c|c|c|c|}
\hline Famílias/Espécies & $\begin{array}{c}\text { DA } \\
\text { (ind.ha }{ }^{-1} \text { ) } \\
\end{array}$ & $\begin{array}{l}\text { DR } \\
(\%)\end{array}$ & $\begin{array}{l}\text { FA } \\
(\%) \\
\end{array}$ & $\begin{array}{l}\text { FR } \\
(\%) \\
\end{array}$ & $\begin{array}{c}\text { DoA } \\
\left(\mathrm{m}^{2} \cdot \mathrm{ha}^{-1}\right)\end{array}$ & $\begin{array}{c}\text { DoR } \\
(\%) \\
\end{array}$ & $\begin{array}{l}\text { VI } \\
(\%) \\
\end{array}$ \\
\hline \multicolumn{8}{|l|}{ Anacardiaceae } \\
\hline Myracrodruon urundeuva Fr. All. & 167,50 & 10,14 & 0,85 & 5,5 & 3,27 & 20,29 & 11,97 \\
\hline Schinopsis brasiliensis Engl. & 132,50 & 8,02 & 0,80 & 5,18 & 1,13 & 7,04 & 6,75 \\
\hline Spondias tuberosa Arruda & $*$ & & & & & & \\
\hline \multicolumn{8}{|l|}{ Apocynaceae } \\
\hline Aspidosperma pyrifolium Mart. & 43,75 & 2,65 & 0,55 & 3,56 & 0,13 & 0,78 & 2,33 \\
\hline \multicolumn{8}{|l|}{ Araliaceae } \\
\hline Aralia excelsa (Griseb.) J.Wen & 1,25 & 0,08 & 0,05 & 0,32 & $<0,01$ & 0,01 & 0,14 \\
\hline \multicolumn{8}{|l|}{ Arecaceae } \\
\hline Syagrus oleracea (Mart.) Becc. & $*$ & & & & & & \\
\hline \multicolumn{8}{|l|}{ Bignoniaceae } \\
\hline Handroanthus impetiginosus (Mart. ex DC.) Mattos & 162,50 & 9,83 & 0,75 & 4,85 & 0,82 & 5,11 & 6,6 \\
\hline
\end{tabular}

Cerne, Lavras, v. 17, n. 2, p. 247-258, abr./jun. 2011 
Tabela 1 - Continua...

Tabela 1 - Continued..

\begin{tabular}{|c|c|c|c|c|c|c|c|}
\hline Famílias/Espécies & $\begin{array}{c}\text { DA } \\
\text { (ind.ha-1) }\end{array}$ & $\begin{array}{l}\mathrm{DR} \\
(\%)\end{array}$ & $\begin{array}{l}\text { FA } \\
(\%)\end{array}$ & $\begin{array}{l}\text { FR } \\
(\%)\end{array}$ & $\begin{array}{c}\text { DoA } \\
\left(\mathrm{m}^{2} \cdot \mathrm{ha}^{-1}\right) \\
\end{array}$ & $\begin{array}{c}\text { DoR } \\
(\%)\end{array}$ & $\begin{array}{l}\text { VI } \\
(\%)\end{array}$ \\
\hline Handroanthus ochraceus (Cham.) Mattos & 6,25 & 0,38 & 0,05 & 0,32 & 0,12 & 0,74 & 0,48 \\
\hline Tabebuia roseoalba (Ridl.) Sand. & 62,50 & 3,78 & 0,60 & 3,88 & 0,29 & 1,83 & 3,16 \\
\hline \multicolumn{8}{|l|}{ Burseraceae } \\
\hline Commiphora leptophloeos (Mart.) Gillett & $*$ & & & & & & \\
\hline \multicolumn{8}{|l|}{ Cactaceae } \\
\hline Cereus jamacaru Hort. Vindob. Ex Salm-Dyck. & * & & & & & & \\
\hline Pereskia bahiensis Guerke & 2,50 & 0,15 & 0,05 & 0,32 & 0,01 & 0,04 & 0,17 \\
\hline \multicolumn{8}{|l|}{ Cannabaceae } \\
\hline Celtis brasiliensis (Gardner) Planch. & $*$ & & & & & & \\
\hline \multicolumn{8}{|l|}{ Celastraceae } \\
\hline Fraunhofera multiflora Mart. & * & & & & & & \\
\hline \multicolumn{8}{|l|}{ Combretaceae } \\
\hline Combretum duarteanum Cambess. & 52,50 & 3,18 & 0,70 & 4,53 & 0,25 & 1,52 & 3,08 \\
\hline \multicolumn{8}{|l|}{ Erythroxyllaceae } \\
\hline Erythroxylum betulaceum Mart. & 3,75 & 0,23 & 0,10 & 0,65 & 0,01 & 0,07 & 0,32 \\
\hline \multicolumn{8}{|l|}{ Euphorbiaceae } \\
\hline Cnidoscolus oligandrus (Müll.Arg.) Pax & 1,25 & 0,08 & 0,05 & 0,32 & 0,01 & 0,09 & 0,16 \\
\hline Sapium glandulosum (L.) Morong & 8,75 & 0,53 & 0,35 & 2,27 & 0,02 & 0,15 & 0,98 \\
\hline \multicolumn{8}{|l|}{ Fabaceae Caesalpinioideae } \\
\hline Apuleia leiocarpa (Vog.) Macbr. & $*$ & & & & & & \\
\hline Centrolobium sclerophyllum H.C.Lima & 10,00 & 0,61 & 0,15 & 0,97 & 0,05 & 0,30 & 0,62 \\
\hline Goniorrhachis marginata Taub. & 3,75 & 0,23 & 0,15 & 0,97 & 0,04 & 0,22 & 0,47 \\
\hline $\begin{array}{l}\text { Senna macranthera (DC. ex Collad.) H. S. Irwin \& } \\
\text { Barneby }\end{array}$ & 5,00 & 0,30 & 0,20 & 1,29 & 0,02 & 0,13 & 0,58 \\
\hline Senna spectabilis (DC.) Irwin et Barn. & $*$ & & & & & & \\
\hline \multicolumn{8}{|l|}{ Fabaceae Cercideae } \\
\hline Bauhinia forficata Link & 5,00 & 0,30 & 0,20 & 1,29 & 0,01 & 0,09 & 0,56 \\
\hline \multicolumn{8}{|l|}{ Fabaceae Faboideae } \\
\hline Machaerium floridum (Mart. ex Benth.) Ducke & $*$ & & & & & & \\
\hline Deguelia nitidula (Benth.) Az.-Tozzi & 5,00 & 0,30 & 0,15 & 0,97 & 0,04 & 0,27 & 0,52 \\
\hline Machaerium hirtum (Vell.) Stellfeld & 15,00 & 0,91 & 0,35 & 2,27 & 0,09 & 0,58 & 1,25 \\
\hline Machaerium acutifolium Vogel & 48,75 & 2,95 & 0,50 & 3,24 & 0,27 & 1,66 & 2,61 \\
\hline Diplotropis ferruginea Benth. & 46,25 & 2,80 & 0,60 & 3,88 & 0,22 & 1,37 & 2,69 \\
\hline Machaerium scleroxylon Tul. & $*$ & & & & & & \\
\hline Dalbergia cearensis Ducke & 6,25 & 0,38 & 0,20 & 1,29 & 0,02 & 0,13 & 0,6 \\
\hline
\end{tabular}

Cerne, Lavras, v. 17, n. 2, p. 247-258, abr./jun. 2011 
Tabela 1 - Continua...

Tabela 1 - Continued..

\begin{tabular}{|c|c|c|c|c|c|c|c|}
\hline Famílias/Espécies & $\begin{array}{c}\text { DA } \\
\text { (ind.ha-1) }\end{array}$ & $\begin{array}{l}\text { DR } \\
(\%)\end{array}$ & $\begin{array}{l}\text { FA } \\
(\%)\end{array}$ & $\begin{array}{l}\text { FR } \\
(\%)\end{array}$ & $\begin{array}{c}\text { DoA } \\
\left(\mathrm{m}^{2} \cdot \mathrm{ha}^{-1}\right)\end{array}$ & $\begin{array}{l}\text { DoR } \\
(\%)\end{array}$ & $\begin{array}{l}\text { VI } \\
(\%)\end{array}$ \\
\hline Machaerium villosum Vogel & 2,50 & 0,15 & 0,10 & 0,65 & 0,01 & 0,03 & 0,28 \\
\hline Platymiscium blanchetii Benth. & 47,5 & 2,87 & 0,65 & 4,21 & 0,52 & 3,20 & 3,43 \\
\hline Platymiscium floribundum Vog. & 2,50 & 0,15 & 0,10 & 0,65 & 0,05 & 0,32 & 0,37 \\
\hline \multicolumn{8}{|l|}{ Fabaceae Mimosoideae } \\
\hline Senegalia polyphylla (DC.) Britton \& Rose & 7,50 & 0,45 & 0,20 & 1,29 & 0,06 & 0,4 & 0,72 \\
\hline Leucochloron limae Barneby \& J.W.Grimes & 75,00 & 4,54 & 0,65 & 4,21 & 0,61 & 3,78 & 4,17 \\
\hline $\begin{array}{l}\text { Blanchetiodendron blanchetii (Benth.) Barneby \& } \\
\text { J.W.Grimes }\end{array}$ & 15,00 & 0,91 & 0,30 & 1,94 & 0,08 & 0,48 & 1,11 \\
\hline Anadenanthera colubrina (Vell.) Brenan & 185,00 & 11,20 & 0,95 & 6,15 & 4,24 & 26,31 & 14,55 \\
\hline Chloroleucon foliolosum (Benth.) G.P.Lewis & 7,50 & 0,45 & 0,15 & 0,97 & 0,13 & 0,80 & 0,74 \\
\hline Enterolobium contortisiliquum (Vell.) Morong. & 1,25 & 0,08 & 0,05 & 0,32 & 0,11 & 0,66 & 0,35 \\
\hline Mimosa tenuiflora (Willd.) Poiret & 3,75 & 0,23 & 0,05 & 0,32 & 0,04 & 0,25 & 0,27 \\
\hline Piptadenia gonoacantha (Mart.) J.F. Macbr. & 45,00 & 2,72 & 0,65 & 4,21 & 0,34 & 2,10 & 3,01 \\
\hline Piptadenia viridiflora (Kunth) Benth. & 3,75 & 0,23 & 0,15 & 0,97 & 0,02 & 0,13 & 0,44 \\
\hline Poincianella pluviosa (DC.) L.P.Queiroz & 275,00 & 16,64 & 1,00 & 6,47 & 2,11 & 13,10 & 12,07 \\
\hline \multicolumn{8}{|l|}{ Lamiaceae } \\
\hline Aegiphila sellowiana Cham. & 1,25 & 0,08 & 0,05 & 0,32 & $<0,01$ & 0,01 & 0,14 \\
\hline Vitex laciniosa Turcz. & 5,00 & 0,30 & 0,10 & 0,65 & 0,01 & 0,05 & 0,33 \\
\hline \multicolumn{8}{|l|}{ Lauraceae } \\
\hline Nectandra nitidula Nees & 1,25 & 0,08 & 0,05 & 0,32 & $<0,01$ & 0,02 & 0,14 \\
\hline \multicolumn{8}{|l|}{ Malvaceae } \\
\hline Cavanillesia arborea K. Schum. & * & & & & & & \\
\hline Ceiba pubiflora (A.St.-Hil.) K.Schum. & * & & & & & & \\
\hline Lueheia paniculata Mart. & 2,50 & 0,15 & 0,10 & 0,65 & $<0,01$ & 0,03 & 0,27 \\
\hline Pseudobombax marginatum (A.St.-Hil.) A.Robyns & 1,25 & 0,08 & 0,05 & 0,32 & 0,09 & 0,55 & 0,32 \\
\hline Sterculia striata St. Hil. et Naud. & $*$ & & & & & & \\
\hline \multicolumn{8}{|l|}{ Meliaceae } \\
\hline Cabralea canjerana (Vell.) Mart. & 3,75 & 0,23 & 0,05 & 0,32 & 0,08 & 0,48 & 0,34 \\
\hline Cedrela fissilis Vell. & * & & & & & & \\
\hline \multicolumn{8}{|l|}{ Moraceae } \\
\hline Maclura tinctoria D. Don ex Steud. & $*$ & & & & & & \\
\hline \multicolumn{8}{|l|}{ Myrtaceae } \\
\hline Eugenia florida DC. & 48,75 & 2,95 & 0,75 & 4,85 & 0,13 & 0,83 & 2,88 \\
\hline \multicolumn{8}{|l|}{ Nyctaginaceae } \\
\hline Guapira opposita Vell. & 2,50 & 0,15 & 0,10 & 0,65 & $<0,01$ & 0,03 & 0,28 \\
\hline
\end{tabular}

Cerne, Lavras, v. 17, n. 2, p. 247-258, abr./jun. 2011 
Tabela 1 - Continua...

Tabela 1 - Continued...

\begin{tabular}{|c|c|c|c|c|c|c|c|}
\hline Famílias/Espécies & $\begin{array}{c}\text { DA } \\
\text { (ind.ha-1) }\end{array}$ & $\begin{array}{l}\mathrm{DR} \\
(\%) \\
\end{array}$ & $\begin{array}{l}\text { FA } \\
(\%) \\
\end{array}$ & $\begin{array}{l}\text { FR } \\
(\%)\end{array}$ & $\begin{array}{c}\text { DoA } \\
\left(\mathrm{m}^{2} \cdot \mathrm{ha}^{-1}\right)\end{array}$ & $\begin{array}{c}\text { DoR } \\
(\%)\end{array}$ & $\begin{array}{c}\text { VI } \\
(\%) \\
\end{array}$ \\
\hline \multicolumn{8}{|l|}{ Polygonaceae } \\
\hline Coccoloba schwackeana Lindau & 15,00 & 0,91 & 0,50 & 3,24 & 0,11 & 0,68 & 1,61 \\
\hline \multicolumn{8}{|l|}{ Rhamnaceae } \\
\hline Ziziphus joazeiro Mart. & * & & & & & & \\
\hline \multicolumn{8}{|l|}{ Rubiaceae } \\
\hline Guettarda angelica Mart. ex Müll.Arg. & 16,25 & 0,98 & 0,25 & 1,62 & 0,05 & 0,33 & 0,98 \\
\hline Randia armata (Sw.) DC. & 31,25 & 1,89 & 0,45 & 2,91 & 0,09 & 0,54 & 1,78 \\
\hline \multicolumn{8}{|l|}{ Rutaceae } \\
\hline Balfourodendron molle (Miq.) Pirani & 57,50 & 3,48 & 0,40 & 2,59 & 0,37 & 2,32 & 2,8 \\
\hline Zanthoxylum riedelianum Engl. & 5,00 & 0,30 & 0,20 & 1,29 & 0,03 & 0,16 & 0,59 \\
\hline \multicolumn{8}{|l|}{ Sapindaceae } \\
\hline Allophylus racemosus $\mathrm{Sw}$. & * & & & & & & \\
\hline Total & 1652,50 & & 15,45 & & 16,10 & & \\
\hline
\end{tabular}

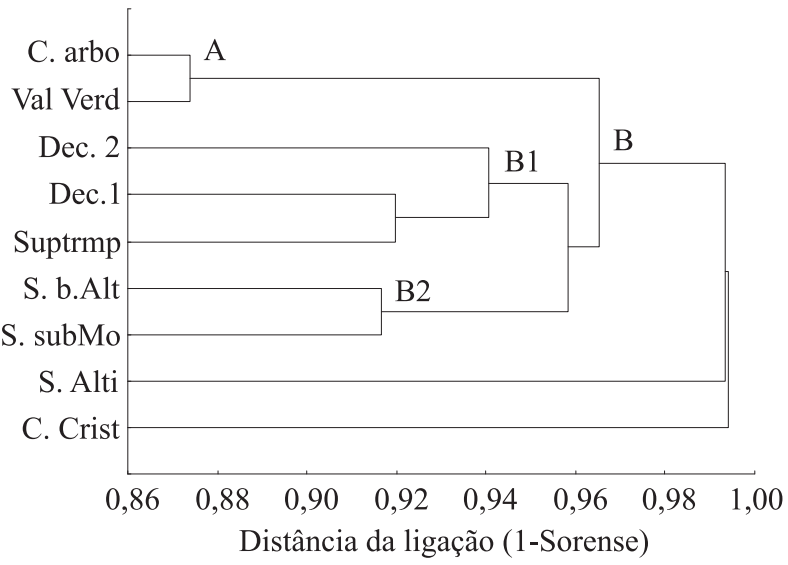

Figura 2 - Dendrograma de dissimilaridade florística entre a área de estudo (Vale Verde) e grupos de espécies indicadoras correspondentes a espécies de ampla distribuição (Suptrmp) e às fitofisionomias: Caatinga Arbórea (C. arbo), Caatinga do Cristalino (C. Crist), Florestas Estacionais Deciduais extraCaatinga (Dec. 1 e Dec. 2), Florestas Estacionais Semideciduais de baixa altitude (S. b.Alt), sub/Montana (S. subMo) e Altimontana (S. Alti).

Figure 2 - Dendrogram of floristic dissimilarity between the study site (Vale Verde) and groups of indicator species corresponding to widely distributed species (Suptrmp) and to the phytophysiognomies: Arboreous Caatinga (C. arbo), Caatinga on Crystalline Basement (C. Crist), Extra-Caatinga Seasonal Deciduous Forests (Dec. 1 and Dec. 2), Submontane (S. subMo), Altimontane (S. Alti) and Low Altitude Seasonal Semideciduous Forests (S. b.Alt)
Com relação à riqueza de espécies, foi encontrado valor semelhante ao de outros remanescentes amostrados na região (SANTOS et al., 2007). Além disso, o número de espécies neste trabalho pode ser considerado dentro da amplitude observada em outros estudos em diferentes áreas de floresta estacional decidual no Brasil (ALCOFORADOFILHO et al., 2003; ARAÚJO et al., 1998a,b; 1995; FERRAZ et al., 1998; IVANAUSKAS; RODRIGUES, 2000; PEREIRA et al., 2002; RODAL et al., 1998, 1999; SILVA; SCARIOT, 2003). Sendo superior ao de Silva e Scariot (2003) que encontraram 36 espécies em um fragmento na bacia do Rio Paranã (GO), e que representa o menor número de espécies encontrado entre as florestas estacionais deciduais, por outro lado, foi inferior as 110 espécies encontradas, na região de Piracicaba (SP), com o maior número de espécies encontradas nas florestas estacionais deciduais (IVANAUSKAS; RODRIGUES, 2000).

Dentre as espécies amostradas, cabe destacar que Anadenanthera colubrina, Machaerium acutifolium, Myracrodruon urundeuva e Aspidosperma pyrifolium, abundantes na área de estudo, são mencionadas na literatura como sendo de ampla distribuição e indicadoras de florestas deciduais e da presença do antigo arco pleistocênico (ARAÚJO; HARIDASAN, 1997; PRADO; GIBBS, 1993). Não obstante, deve-se ressaltar a presença de taxas frequentes nas florestas estacionais deciduais nordestinas, como Schinopsis brasiliensis, Spondias tuberosa (Anacardiaceae), Cereus jamacaru (Cactaceae), 
Piptadenia viridiflora (Fabaceae) e Ziziphus joazeiro (Rhamnaceae) (SALES et al., 1998).

Estrutura - Foram amostrados 1.322 indivíduos, pertencentes a 47 espécies arbóreas, a 42 gêneros e a 19 famílias. A densidade e a dominância absolutas, estimadas, foram de 1.652,5 ind.ha ${ }^{-1}$ e $16,10 \mathrm{~m}^{2} . \mathrm{ha}^{-1}$, respectivamente (Tabela 1). Nesse sentido, a densidade encontrada no presente estudo foi superior aos valores considerados por Nascimento et al. (2004) como dentro dos padrões para vegetações deciduais extra-Caatinga.

Por outro lado, a área basal foi inferior aos valores encontrados em outros trabalhos realizados nas florestas deciduais (NASCIMENTO et al., 2004). Dessa forma, é provável que características regionais de clima e solo possam ter favorecido o elevado número de indivíduos de pequeno porte, muitas vezes bifurcados e/ou ocorrendo de forma agregada, o que pode ser observado pela baixa frequência das espécies (Tabela 1).

Cerca de $81 \%$ dos indivíduos amostrados na estrutura corresponderam a apenas três das 19 famílias. A família melhor representada foi Fabaceae, com 49\% dos indivíduos, cujo destaque deve-se principalmente às espécies Poincianella pluviosa (220 indivíduos) e Anadenanthera colubrina (148 indivíduos), seguida por Anacardiaceae com 240 indivíduos, representada por apenas duas espécies no levantamento, Myracrodruon urundeuva e Schinopsis brasiliensis com 134 e 106 indivíduos, respectivamente. A família Bignoniaceae contribuiu com 185 indivíduos, com destaque para Handroanthus impetiginosus com 130 indivíduos. Juntas, estas famílias representaram 56\% dos indivíduos amostrados.

As dez espécies amostradas com maior VI representaram cerca de $68,8 \%$ do VI total da comunidade (Tabela 1). Dentre elas, A. colubrina, P. pluviosa, M. urundeuva, S. brasiliensis e H. impetiginosus apresentaram os maiores valores de importância (VI), somando 51,9\% do VI total. Dessas, A. colubrina e M. urundeuva apresentaram alguns indivíduos com grandes diâmetros, associados à elevada densidade e frequência das espécies. Já P. pluviosa, S. brasiliensis e H. impetiginosus destacaram-se pela densidade e frequencia. A dominância relativa das espécies A. colubrina e $M$. urundeuva, em florestas decíduas, é maior que a encontrada em florestas de maior umidade, ou seja, provavelmente há um alto grau de exclusão competitiva por parte destas espécies (FAGUNDES et al., 2007), favorecidas pelas características ambientais como clima e solo, que confere a elas a característica de boas indicadoras de solos mesotróficos (SALIS et al., 2004).
Poincianella pluviosa e S. brasiliensis estão entre as espécies frequentemente encontradas nas listas de estudos realizados na caatinga (SAMPAIO, 1996). Já $H$. impetiginosus é uma espécie muito frequente e representativa tanto em área basal quanto em densidade nos levantamentos em florestas estacionais deciduais fora deste bioma (NASCIMENTO et al., 2004; SILVA; SCARIOT, 2003), o que pode ser resultado da posição geográfica da área de estudo.

O fato de a área estudada estar localizada em ambiente de transição ecológica entre Caatinga/Cerrado justifica também a ocorrência mútua de espécies típicas da Caatinga como Poincianella pluviosa, Cavanillesia arborea, Cereus jamacaru, Cnidosculus pubescens, Combretum duarteanum entre outras, e espécies de ampla ocorrência e que são normalmente encontradas em formações do Cerrado, como Handroanthus ochraceus, Erythroxylum deciduum, Eugenia florida, Zanthoxylum ridelianum, Guapira opposita, entre outras. Por outro lado, é possível observar que, embora a posição geográfica do remanescente permita a ocorrência de espécies alóctones à sua fitofisionomia, estas espécies constituem uma pequena proporção do contingente da comunidade, o que pode ser expresso pelos baixos VIs estimados para as mesmas (Tabela 1).

Com relação à distribuição dos indivíduos em classes de diâmetro, tanto as espécies mais abundantes, A. colubrina, P. pluviosa e M. urundeuva, assim como a comunidade como um todo, apresentaram padrões semelhantes (Figura 3 a, b, c e d). Neste sentido, foram observadas menores freqüências de indivíduos nas classes menores e maiores, caracterizando uma distribuição semelhante à normal.

Considerando que a distribuição diamétrica pode refletir o histórico da floresta, bem como a ocorrência de distúrbios passados como fogo, corte seletivo e herbivoria (MEYER et al., 1961), o padrão encontrado pode estar relacionado a fatores antrópicos que podem estar interferindo na regeneração natural da área de estudo (SANTOS; VIEIRA, 2005). Mais especificamente, a herbivoria por animais domesticados está associada à redução do recrutamento, do crescimento e da distribuição geográfica de várias espécies de plantas herbáceas, arbustivas e arbóreas (PEREVOLOTSKY; HAIMOV, 1992; OBA, 1998; SEVERSON; DEBANO, 1991). Além disso, existem evidências de que a herbivoria por animais domésticos pode afetar a estrutura e a capacidade de regeneração da vegetação (PEREVOLOTTSKY; HAIMOV, 1992). 

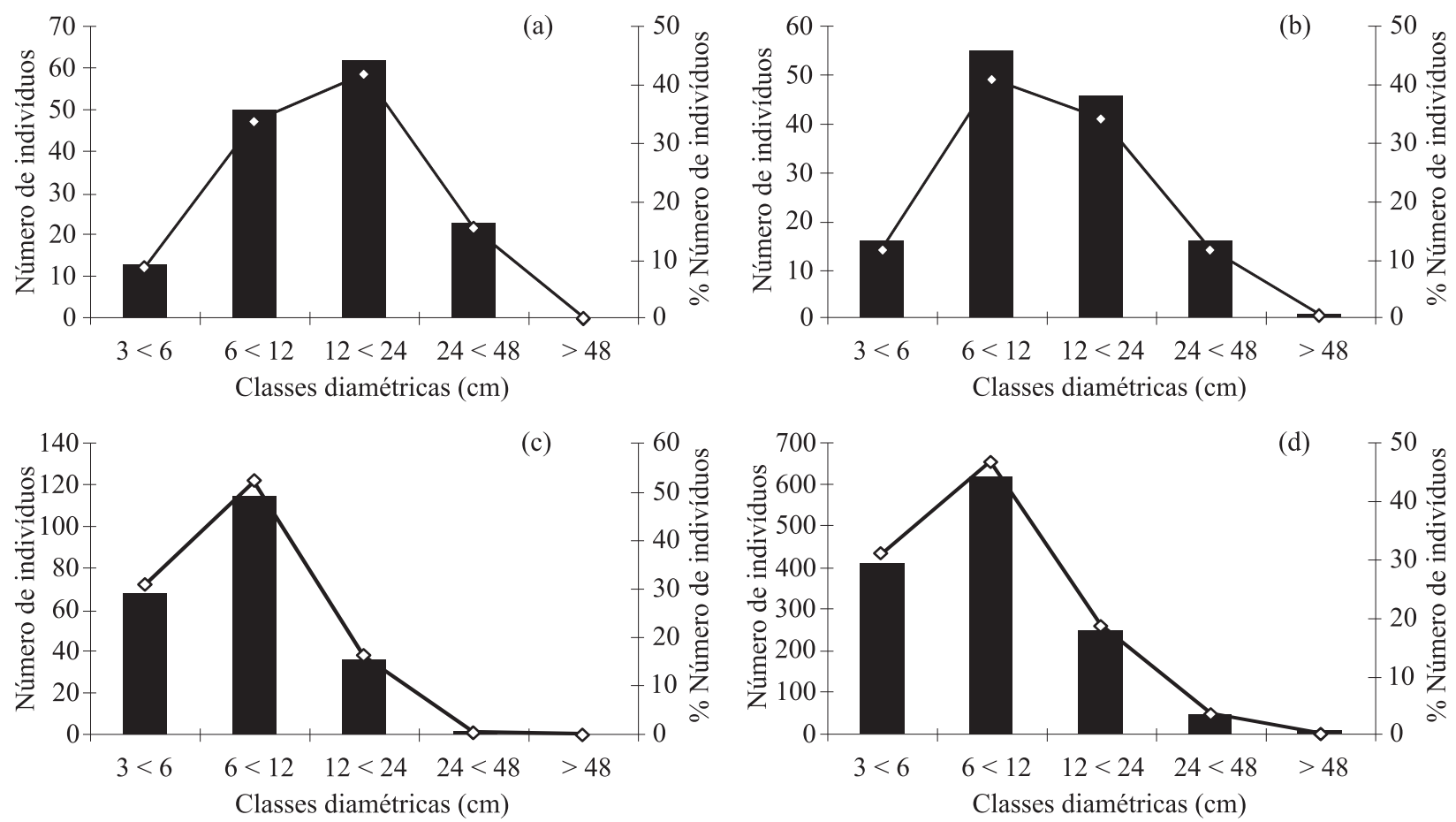

Número de indivíduos

\% Número de indivíduos

Figura 3 - Distribuição diamétrica das populações com maiores valores de importância: Anadenanthera colubrina (a), Poincianella pluviosa (b), Myracrodruon urundeuva (c) e da comunidade arbóreas (d), amostradas no fragmento Vale Verde, município de Juvenília, Minas Gerais.

Figure 3 -Diameter distribution of populations with greater importance values: Anadenanthera colubrina (a), Poincianella pluviosa (b), Myracrodruon urundeuva (c) and of the arboreuos community (d), as sampled in the 'Vale Verde'fragment, in Juvenilia, Minas Gerais.

Por outro lado, o padrão de distribuição diamétrica diferente do "J-reverso" não é um evento incomum para as espécies $A$. colubrina e $M$. urundeuva, o que indica que este possa estar ligado à própria ecologia das espécies (FAGUNDES et al., 2007; FELFILI, 1995; NASCIMENTO et al., 2004; SOUZA et al., 1994). Neste sentido, é provável que efeitos antrópicos que ocorreram no passado e que ainda estão presentes modificaram a estrutura da floresta, todavia, são necessários trabalhos visando à dinâmica da comunidade associados a ações de conservação do remanescente, para testar a influência dos fatores biológicos na estrutura da comunidade.

O índice de diversidade de Shannon $\left(H^{\prime}\right)$ foi estimado em 2,94 nats.ind ${ }^{-1}$ e a equabilidade $\left(J^{\prime}\right)$ em 0,76 . Comparando a diversidade estimada neste trabalho com a encontrada em outras florestas estacionais deciduais pelo Brasil, o índice de Shannon foi bem similar ao valor encontrado $\left(\mathrm{H}^{\prime}=2,99\right.$ nats.ind $\mathrm{d}^{-1}$ ) em 0,6 ha (DNS $\geq 3 \mathrm{~cm}$ ), no agreste paraibano
(PEREIRA et al., 2002). Também foi semelhante ao obtido no estudo de Ivanauskas e Rodrigues (2000), H' $=3,00$ nats.ind ${ }^{-1}$ com indivíduos de DAP $\geq 5 \mathrm{~cm}$ encontrado em uma amostra de 0,43 ha em Piracicaba, SP e Silva e Scariot (2003), em um censo dos indivíduos com DAP $\geq 5 \mathrm{~cm}$ em São Domingos, GO, obtiveram 2,99 nats.ind ${ }^{-1}$.

No entanto, o valor encontrado é superior àqueles registrados em áreas de Caatinga xerófila $-\mathrm{H}^{\prime}=1,64$ a 2,54 nats.ind ${ }^{-1}$ (ARAÚJO et al., 1995; FERRAZ et al., 1998; RODAL et al., 1999). Os baixos valores de diversidade de espécies encontrados nas áreas de Caatinga xerófilas podem estar associados, dentre outros fatores, às características bioclimáticas inerentes à própria fitofisionomia (ARAÚJO et al., 1995), que podem restringir o desenvolvimento da vegetação. Por outro lado, o incremento de espécies não deciduais na amostragem, devido ao caráter ecotonal da região de estudo, pode ter sido responsável pelo índice de diversidade estimado. 
Já o valor da equabilidade estimada $\left(\mathrm{J}^{\prime}=0,76\right)$ indica distribuição desigual dos indivíduos amostrados entre as espécies da comunidade, ou seja, há grande dominância ecológica na área de estudo, fato que pode ser confirmado pela alta densidade e dominância de algumas espécies (ver Tabela 1). Todavia, convém ressaltar que o predomínio em número ou massa de poucas espécies em uma comunidade, não é incomum em florestas tropicais (RICHARDS, 1952), particularmente entre as florestas estacionais do Brasil Central (OLIVEIRA-FILHO; RATTER, 2000).

Foi possível observar, no remanescente estudado, que embora haja interferências humanas evidentes na área e que estas podem ter comprometido a regeneração natural, o fragmento mantém suas características florísticas semelhantes à encontrada em outras formações de florestas estacionais deciduais. Por outro lado, a carência científica com relação às espécies presentes neste tipo de fitofisionomia evidencia a necessidade de novos trabalhos visando conhecer a estrutura e composição de espécies destes ambientes, bem como trabalhos de dinâmica, para que seja possível diferenciar com clareza os efeitos antrópicos das respostas naturais ecológicas, próprias da comunidade.

\section{REFERÊNCIAS}

AB'SABER, A. Os domínios de natureza no Brasil. 3. ed. São Paulo: Ateliê Editorial, 2005. 159p.

ALCOFORADO-FILHO, F. G.; SAMPAIO, E. V. S. B.; RODAL, M. J. N. Florística e fitossociologia de um remanescente de vegetação caducifólia espinhosa arbórea em Caruaru, Pernambuco. Acta Botanica Brasílica, São Paulo, v. 17, n. 2, p. 287-303, jun. 2003.

ALMEIDA, H. S.; MACHADO, E. L. M. Espécies indicadoras do componente arbóreo em comunidades de Floresta Estacional Decídua. Revista Brasileira de Biociências, Porto Alegre, v. 5, n. 1, p. 654-656, jul. 2007.

ANGIOSPERM PHYLOGENY GROUP, THE. An update of the Angiosperm Phylogeny Group classification for the orders and families of flowering plants: APG II. Botanical Journal of the Linnean Society, v. 141, p. 399-436, 2003. Disponível em: <http://www.blackwell-synergy.com/links/ doi/10.1046/j.1095-8339.2003.t01-1-00158.x/pdf>. Acesso em: 14 jan. 2008.

ARAÚJO, F. S.; SAMPAIO, E. V. S. B.; FIGUEIREDO, M. A.; RODAL, M. J. N.; FERNANDES, A. G. Composição

Cerne, Lavras, v. 17, n. 2, p. 247-258, abr./jun. 2011 florística da vegetação de carrasco, Novo Oriente, CE. Revista Brasileira de Botânica, São Paulo, v. 21, n. 2, p. 105-116, abr. 1998a.

ARAÚJO, E. L.; SAMPAIO, E. V. S. B.; RODAL, M. J. N. Composição florística e fitossociológica de três áreas de Caatinga de Pernambuco. Revista Brasileira de Biologia, São Carlos, v. 55, n. 4, p. 595-607, out. 1995.

ARAÚJO, F. S.; SAMPAIO, E. V. S. B.; RODAL, M. J. N.; FIGUEIREDO, M. A. Organização comunitária do componente lenhoso de três áreas de carrasco em Novo Oriente, CE. Revista Brasileira de Biologia, São Carlos, v. 58, n. 1, p. 85-95, jan. 1998 b.

ARAÚJO, G. M.; HARIDASAN, M. Estrutura fitossociológica de duas matas mesófilas semidecíduas em Uberlândia, Triângulo Mineiro. Naturalia, Rio Claro, v. 22, p. 115-129, jan. 1997.

BOTREL, R. T.; OLIVEIRA-FILHO, A. T.; RODRIGUES, L. A.; CURI, N. Composição florística e estrutura da comunidade arbórea de um fragmento de Floresta Estacional Semidecidual em Ingaí-MG, e a influência de variáveis ambientais na distribuição das espécies. Revista Brasileira de Botânica, São Paulo, v. 25, n. 2, p. 195-213, jun. 2002.

\section{EMPRESA BRASILEIRA DE PESQUISA}

AGROPECUÁRIA. Manual de métodos de análises de solo. 2. ed. Rio de Janeiro, 1997. 412 p.

FAGUNDES, L. M.; CARVALHO, D. A.; BERG, E. van den; MARQUES, J. J. G. S. M.; MACHADO, E. L. M. Florística e estrutura do estrato arbóreo de dois fragmentos de florestas deciduais às margens do rio Grande, em Alpinópolis e Passos, MG, Brasil. Acta Botanica Brasilica, São Paulo, v. 21, n. 1, p. $65-78$, jan. 2007.

FELFILI, J. M. Diversity, structure and dynamics of a gallery forest in central Brazil. Vegetatio, v. 117, n. 1, p. 1-15, Jan. 1995.

FERNANDES, A. Conexões florísticas do Brasil. Fortaleza: Banco do Nordeste, 2003. 135 p.

FERRAZ, E. M. N.; RODAL, M. J. N.; SAMPAIO, E. V. S. B.; PEREIRA, R. C. A. Composição florística em trechos de vegetação de caatinga e brejo de altitude na região do Vale do Pajeú, Pernambuco. Revista Brasileira de Botânica, São Paulo, v. 21, n. 1, p. 7-15, jan. 1998. 
IVANAUSKAS, N. M.; RODRIGUES, R. R. Florística e fitossociologia de remanescentes de floresta estacional decidual em Piracicaba, São Paulo, Brasil. Revista Brasileira de Botânica, São Paulo, v. 23, n. 3, p. 291-304, jul. 2000.

KENT, M.; COKER, P. Vegetation description and analysis: a practical approach. London: Belhaven, 1992. 363 p.

MARTINS, F. R. Estrutura de uma floresta mesófila. 2. ed. Campinas: Unicamp, 1993. 246 p.

MEYER, H. A.; RECKNAGEL, A. B.; STEVENSON, D. D.; BARTOO, R. A. Forest management. 2. ed. New York: Ronald, 1961. 282 p.

MUELLER-DOMBOIS, D.; ELLEMBERG, H. Aims and methods of vegetation ecology. New York: J. Wiley, 1974. 547 p.

NASCIMENTO, A. R. T.; FELFILI, J. M.; MEIRELLES, E. M. Florística e estrutura da comunidade arbórea de um remanescente de floresta estacional decidual de encosta, Monte Alegre, GO, Brasil. Acta Botanica Brasílica, São Paulo, v. 18, n. 3, p. 659-669, jul. 2004.

OBA, G. Effects of excluding goat herbivory on Acacia tortilis woodland around pastoralist settlements in northwest Kenya. Acta Oecologica, v. 19, n. 4, p. 395-404, 1998. Disponível em: $<$ http http://www.sciencedirect.com/science?_ob=ArticleList URL\&_method=list\&_ArticleListID $=1001189596 \&$ sort $=$ r\&view $=$ c\&_acct $=$ C000037521\&_version $=1 \&$ urlVersion $=0 \&$ userid $=686380 \& \mathrm{md} 5=985 \mathrm{c} 76 \mathrm{a} 0 \mathrm{bad} 46 \mathrm{e} 1 \mathrm{c} 18 \mathrm{~d} 3$ 46de9 26edd6>. Acesso em: 4 set. 2009.

\section{OLIVEIRA-FILHO, A. T.; CURI, N.; VILELA, E. A.;} CARVALHO, D. A. Effects of canopy gaps, topography and soils on the distribution of woody species in a central brasilian deciduous dry forest. Biotropica, v. 30, p. 362-375, 1998. Disponível em: <http://www.icb.ufmg.br/treeatlan/>. Acesso em: 4 set. 2009.

OLIVEIRA-FILHO, A. T.; FONTES, M. A. Patterns of floristic differentiation among Atlantic Forests in southeastern Brazil and the influence of climate. Biotropica, v. 32, n. 4b, p. 793-810, Sept. 2000. Disponível em: <http://www.icb.ufmg. br/treeatlan>. Acesso em: 1 mar. 2011.

OLIVEIRA-FILHO, A. T.; RATTER, J. A. Padrões florísticos das matas ciliares da região dos cerrados e a evolução das paisagens do Brasil Central durante o quaternário tardio. In: RODRIGUES, R. R.; LEITÃO-FILHO, H. F. Matas ciliares: conservação e recuperação. São Paulo: Edusp, 2000. p. 73-89.

PEREIRA, I. M.; ANDRADE, L. A.; BARBOSA, M. R. V.; SAMPAIO, E. V. S. B. Composição florística e análise fitossociológica do componente arbustivo-arbóreo de um remanescente florestal no agreste Paraibano. Acta Botânica Brasílica, São Paulo, v. 16, n. 3, p. 357-369, jul. 2002.

PEREVOLOTSKY, A.; HAIMOV, Y. The effect of thinning and goat browsing on the structure and development of mediterranean woodland in Israel. Forest Ecology and Management, Madison, v. 49, p. 61-74, May 1992. Disponível em: <http://www.sciencedirect.com/ science?_ob=ArticleURL\&_udi=B6T6X-49159C3-CF\&_ user $=686380 \&$ _coverDate $=05 \% 2 \mathrm{~F} 31 \% 2 \mathrm{~F} 1992 \& \_$rdoc $=5 \&$ fmt=high\&_orig=browse\&_srch $=$ docinfo $(\% 23$ toc $\% 235042$ \%231992\%23999509998\%23439939\%23FLP\%23display\% 23 Volume)\&_cdi $=5042 \&$ _sort $=\mathrm{d} \&$ docanchor $=\&$ \& ct $=20 \&$ acct $=$ C000037521\&_version $=1 \&$ _urlVersion $=0$ \&_userid $=68$ 6380\&md5=b003bb697d97378d89787a848b53c598>. Acesso em: 4 set. 2009.

PRADO, D. E. As caatingas da América do Sul. In: LEAL, I. L.; TABARELLI, M.; SILVA, J. M. C. Ecologia e conservação da Caatinga. Recife: UFPE, 2003. p. 3-74.

PRADO, D. E.; GIBBS, P. E. Patterns of species distributions in the dry seasonal forest of South America. Annual of the Missouri Botanical Garden, Saint Louis, v. 80, p. 902-927, 1993.

QUEIROZ, L. P. The Brazilian Caatinga: phytogeographical patterns inferred from distribution data of the Leguminosae. In: PENNINGTON, R. T.; RATTER, J. A.; LEWIS, G. P. Neotropical savannas and dry forests: plant diversity, biogeography and conservation. Boca Raton: CRC, 2006. p. 113149. (The Systematics Association Special volume Series, 69).

RICHARDS, P. W. The tropical rain forest. Cambridge: Cambridge University, 1952. 450 p.

RODAL, M. J. N.; ANDRADE, K. V. de S. A.; SALES, M. F. de; GOMES, A. P. S. Fitossociologia do componente lenhoso de um refúgio vegetacional no município de Buíque, Pernambuco. Revista Brasileira de Biologia, São Paulo, v. 58, n. 3, p. 517-526, jul. 1998.

Cerne, Lavras, v. 17, n. 2, p. 247-258, abr./jun. 2011 
RODAL, M. J. N.; BARBOSA, R. V.; THOMAS, W. W. Do the seasonal forests in northeastern Brazil represent a single floristic unit? Brazilian Journal of Biology, São Carlos, v. 68, n. 3, p. 467-475, Aug. 2008.

RODAL, M. J. N.; NASCIMENTO, L. M.; MELO, A. L. Composição florística de um trecho de vegetação arbustiva caducifólia, no município de Ibimirim, PE, Brasil. Acta Botânica Brasílica, São Paulo, v. 13, n. 1, p. 15-28, jan. 1999.

SALES, M. F.; MAYO, S. J.; RODAL, M. J. N. Plantas vasculares das florestas serranas de Pernambuco: um checklist da flora ameaçada dos brejos de altitude. Recife: UFPE, 1998. $130 \mathrm{p}$.

SALIS, S. M.; SILVA, M. P.; MATTOS, P. V.; SILVA, J. S. V.; POTT, V. J.; POTT, A. Fitossociologia de remanescentes de Floresta Estacional Decidual em Corumbá, estado de Mato Grosso do Sul, Brasil. Revista Brasileira de Botânica, São Paulo, v. 27, n. 4, p. 671-684, out. 2004.

SAMPAIO, E. V. S. B. Fitossociologia. In: SAMPAIO, E. V. S. B.; MAYO, S. J.; BARBOSA, M. R. V. Pesquisa botânica nordestina: progresso e perspectivas. Recife: Sociedade Botânica do Brasil, 1996. p. 203-230.

SAMPAIO, E. V. S. B. Overview of the Brazilian Caatinga. In: BULLOCK, S. H.; MOONEY, H. A.; MEDINA, E. Seasonally dry tropical forests. Cambridge: Cambridge University, 1995. p. 35-63.

SANO, S. M.; ALMEIDA, S. P. Cerrado: ambiente e flora. Planaltina: Embrapa-CPAC, 1998. 556 p.

SANTOS, R. M. Identidade e relações florísticas da caatinga arbórea do norte de Minas Gerais e sudeste da Bahia. 2009. 85 p. Tese (Doutorado em Ciências Florestais) Universidade Federal de Lavras, Lavras, 2009.
SANTOS, R. M.; VIEIRA, F. A. Análise estrutural do componente arbóreo de três áreas de cerrado em diferentes estádios de conservação no município de Três Marias, Minas gerais, Brasil. Cerne, Lavras, v. 11, n. 4, p. 399-408, dez. 2005.

SANTOS, R. M.; VIEIRA, F. A.; FAGUNDES, M.; NUNES, Y. R. F.; GUSMÃO, E. Riqueza e similaridade florística de oito remanescentes florestais no norte de Minas Gerais, Brasil. Revista Árvore, Viçosa, v. 31, n. 1, p. 135-144, jan. 2007.

SCOLFORO, J. R. S.; MELLO, J. M. Inventário florestal. Lavras: UFLA/FAEPE, 1997. 341 p.

SEVERSON, K. E.; DEBANO, L. F. Influence of spanish goats on vegetation and soils in Arizona chaparral. Journal of Range Management, Tucson, v. 44, n. 2, p. 111-117, Apr. 1991.

SILVA, L. A.; SCARIOT, A. Composição florística e estrutura da comunidade arbórea em uma floresta estacional decidual em afloramento calcário: fazenda São José, São Domingos, GO, Bacia do Rio Paranã. Acta Botanica Brasilica, São Paulo, v. 17, n. 2, p. 305-313, abr. 2003.

SOUZA, M. J. N.; MARTINS, M. L. R.; SOARES, Z. M. L.; FREITAS-FILHO, M. R.; ALMEIDA, M. A. G.; PINHEIRO, F. S. A.; SAMPAIO, M. A. B.; CARVALHO, G. M. B. S.; SOARES, A. M. L.; GOMES, E. C. B.; SILVA, R. A. Redimensionamento da região semi-árida do Nordeste do Brasil. In: CONFERÊNCIA NACIONAL E SEMINÁRIO LATINO-AMERICANO DE DESERTIFICAÇÃO, 1994, Fortaleza. Anais... Fortaleza: Fundação Esquel do Brasil, 1994.

TABARELLI, T.; SILVA, J. M. C. Áreas e ações prioritárias para a conservação da biodiversidade da caatinga. In: LEAL, I. R.; TABARELLI, M.; SILVA, J. M. C.; BARROS, M. L. B. Ecologia e conservação da caatinga. Recife: UFPE, 2003. p. 777-796. 